\title{
THE MODULE OF PSEUDO-RATIONAL RELATIONS OF A QUOTIENT DIVISIBLE GROUP
}

\author{
A. V. TSAREV
}

\begin{abstract}
It is proved that the module of pseudo-rational relations determines a quotient divisible group up to quasi-isomorphism.
\end{abstract}

\section{INTRODUCTION}

In the most general case, an Abelian group $G$ may be called quotient divisible if it contains a free subgroup $F$ such that $G / F$ is a divisible periodic group. In the present paper, we consider only quotient divisible torsion-free groups of finite rank that do not contain divisible periodic subgroups. Fomin and Wickless introduced such groups in 11; moreover, they constructed the category of quotient divisible groups with quasihomomorphisms as morphisms and showed that this category is dual to the well-known category of torsion-free groups of finite rank with quasihomomorphisms as morphisms.

Even earlier, in [2, Beaumont and Pierce considered quotient divisible torsion-free groups of finite rank (the term itself "quotient divisible group" first appeared in that paper), for which they constructed a system of invariants that determine such a group up to quasi-isomorphism. It was an initial example of a description of Abelian groups up to quasi-isomorphism rather than up to isomorphism, which was quite timely in view of the papers $[3$ by Jónsson and 4 by Yakovlev.

In the present paper, we develop an idea of Fomin from [5] about the invocation of modules over the ring $R$ of pseudo-rational numbers for the study of quotient divisible groups. The point is that any quotient divisible group can be embedded in a certain finitely generated $R$-module, the structure of which is simpler than that of the group itself but still carries much information about the group.

The main results of the paper are Theorems [6 and 7, in which we prove that the module of pseudo-rational relations determines a quotient divisible group up to quasiisomorphism. In a sense, the results obtained here may be regarded as a generalization of the results of Beaumont and Pierce on a description of quotient divisible groups up to quasi-isomorphism.

Throughout the paper, by a "group" we mean an Abelian group written additively; by $\mathbb{Z}, \mathbb{Q}$, and $\widehat{\mathbb{Z}}_{p}$ we denote the ring of integers, the ring of rational numbers, and the ring of $p$-adic integers, respectively, or their additive groups; $\mathbb{Z}(m)$ is the ring (or additive group) of residue classes modulo $m, P$ is the set of all prime numbers, and $\mathbb{N}$ is the set of all natural numbers. If $S$ is a subset of a $K$-module $M$, then by $\langle S\rangle$ and $\langle S\rangle_{K}$ we denote, respectively, the subgroup and the submodule generated by $S ;\langle S\rangle_{*}$ denotes the pure subgroup generated by $S$, which consists of all $r \in M$ such that $n r \in\langle S\rangle$ for some natural $n$.

2010 Mathematics Subject Classification. Primary 20K99.

Key words and phrases. Abelian group, quotient divisible group, torsion-free group of finite rank, ring of pseudo-rational numbers. 
The other notions and notation used in the paper are standard and fully correspond to [6].

\section{§1. Modules over the RING of PSEUdo-Rational Numbers}

Let $\chi=\left(m_{p}\right)$ be an arbitrary characteristic, and let $K_{p}=\mathbb{Z} / p^{m_{p}} \mathbb{Z}$ or $K_{p}=\widehat{\mathbb{Z}}_{p}$ for $m_{p}<\infty$ and $m_{p}=\infty$, respectively. If $\chi$ contains infinitely many nonzero elements, then we consider the ring $\mathbb{Z}_{\chi}=\prod_{p \in P} K_{p}$, in which we construct the ring $R_{\chi}$ that is purely generated by the unity of the ring and by the ideal $\bigoplus_{p \in P} K_{p}$,

$$
R_{\chi}=\left\langle 1, \bigoplus_{p \in P} K_{p}\right\rangle_{*} \subset \prod_{p \in P} K_{p}
$$

If all $p$-components of $\chi$ except for $p_{1}, \ldots, p_{n}$ are equal to zero, then we construct the rings $K_{\chi}=K_{p_{1}} \oplus \ldots \oplus K_{p_{n}}$ and $R_{\chi}=\mathbb{Q} \oplus K_{\chi}$.

Definition 1. The ring $R_{\rho}$, where $\rho=(\infty, \infty, \ldots)$ is the characteristic of the group $\mathbb{Q}$, is called the ring of pseudo-rational numbers and is denoted simply by $R$.

Consider some properties of the ring of pseudo-rational numbers.

1. An element $r=\left(\alpha_{p}\right) \in \prod_{p \in P} \widehat{\mathbb{Z}}_{p}$ belongs to $R$ if and only if there exists a rational number $|r|=\frac{m}{n}$ such that $n \alpha_{p}=m$ for almost all primes $p$.

2. Denote by $\varepsilon_{p}$ the element in $R$ the $p$-component of which is equal to 1 and the other components are zero. Then $\varepsilon_{p}$ is an idempotent of the ring $R$, and consequently, $R=\varepsilon_{p} R \oplus\left(1-\varepsilon_{p}\right) R$.

3. The elements of the form

$$
\varepsilon=\varepsilon_{p_{1}}+\cdots+\varepsilon_{p_{n}},
$$

where $p_{1}, \ldots, p_{n}$ are distinct prime numbers, and the elements of the form

$$
1-\varepsilon,
$$

as well as 1 and 0 , form the set of all idempotents of the ring $R$.

4. Any element $r \in R$ can be expressed as $r=\varepsilon r+(1-\varepsilon)|r|$, where $\varepsilon$ is an idempotent of the form (a).

5. A ring $K$ is an epimorphic image of the ring $R$ if and only if $K \cong R_{\chi}$ or $K \cong K_{\chi}$.

6. The set $T=\bigoplus_{p \in P} \widehat{\mathbb{Z}}_{p}$ is a maximal ideal of the $\operatorname{ring} R$, and $R / T \cong \mathbb{Q}$.

7. The ring of pseudo-rational numbers is hereditary.

Let $M$ be an arbitrary module over the ring of pseudo-rational numbers. Since $R / T \cong$ $\mathbb{Q}$, it follows that the quotient module $M / T M$ is a vector space over $\mathbb{Q}$.

Definition 2. The quantity $\operatorname{dim}_{\mathbb{Q}} M / T M$ is called the pseudo-rational rank of the $R$ module $M$ and is denoted by $r^{*}(M)$.

This invariant was introduced by Fomin. In the theory of $R$-modules, it is as important as the usual rank in the theory of Abelian groups.

8. If $M=\left\langle x_{1}, \ldots, x_{n}\right\rangle_{R}$, then $r^{*}(M) \leq n$.

9. For a quotient module $M / L$, we have

$$
r^{*}(M)=r^{*}(M / L)+r^{*}(L)
$$

An $R$-module $M$ is said to be divisible if the structure of the $R$-module on $M$ coincides with that of a $\mathbb{Q}$-space; i.e., the additive group of the $R$-module $M$ is a torsion-free divisible group and $r \cdot m=|r| \cdot m$ for any $r \in R$ and $m \in M$. An $R$-module $M$ is said to be reduced if it contains no divisible submodules. 
Let $M$ be an arbitrary finitely generated $R$-module with a system of generators $\left\{x_{1}, \ldots, x_{n}\right\}$. Then, obviously, the $\widehat{\mathbb{Z}}_{p}$-module $M_{p}=\varepsilon_{p} M$ is generated by the elements $\left\{\varepsilon_{p} x_{1}, \ldots, \varepsilon_{p} x_{n}\right\}$. The finitely generated $p$-adic module $M_{p}$ is representable as a direct sum of cyclic $\widehat{\mathbb{Z}}_{p}$-modules,

$$
M_{p}=\left\langle a_{1}\right\rangle_{\widehat{\mathbb{Z}}_{p}} \oplus \cdots \oplus\left\langle a_{n}\right\rangle_{\widehat{\mathbb{Z}}_{p}},
$$

where some summands may be zero.

A cyclic $\widehat{\mathbb{Z}}_{p}$-module is isomorphic either to $\mathbb{Z} / p^{k_{i p}} \mathbb{Z}$, where $k_{i p}$ is a nonnegative integer, or to $\widehat{\mathbb{Z}}_{p}$. Consequently, the isomorphism

$$
M_{p} \cong \mathbb{Z}\left(p^{k_{p 1}}\right) \oplus \cdots \oplus \mathbb{Z}\left(p^{k_{p t}}\right) \oplus \bigoplus_{s} \widehat{\mathbb{Z}}_{p}, \quad t+s=n,
$$

determines the following ordered sequence of nonnegative integers and symbols $\infty$ :

$$
0 \leq k_{p 1} \leq \cdots \leq k_{p n} \leq \infty,
$$

where the last $s$ terms are the symbols $\infty(0 \leq s \leq n)$. The sequence (1) over all primes $p$ determines a sequence of characteristics (among which zero characteristics may occur):

$$
\chi_{1} \leq \cdots \leq \chi_{n} .
$$

The sequence (2) is called the generalized cocharacteristic of a finitely generated $R$-module $M$.

\section{§2. Quotient Divisible Groups}

Definition 3. A group $G$ is said to be quotient divisible if it contains no periodic divisible subgroups, but it contains a free subgroup $F$ of finite rank such that $G / F$ is a periodic divisible group.

The free subgroup $F$ in Definition 3 is called a fundamental subgroup of $G$, and any base of it is called a fundamental system of the group.

Let $G$ be a reduced quotient divisible group. Consider its $\mathbb{Z}$-adic completion $\widehat{G}$. The canonical homomorphism $\alpha: G \rightarrow \widehat{G}$ is a monomorphism, because ker $\alpha=\bigcap_{n \in \mathbb{N}} n G=$ $G^{1}=0$. The group $\widehat{G}$ is a $\widehat{\mathbb{Z}}$-module and, thus, a module over the ring of pseudo-rational numbers.

Definition 4. The $R$-module $\mathcal{R}(G)=\operatorname{div} G \oplus\langle\alpha(G)\rangle_{R}$ is called the pseudo-rational envelope (or the pseudo-rational type) of a quotient divisible group $G$.

The pseudo-rational rank of a quotient divisible group is defined to be the pseudorational rank of its pseudo-rational envelope and is denoted by $r^{*}(G)$,

$$
r^{*}(G)=r^{*}(\mathcal{R}(G)) \text {. }
$$

By the generalized cocharacteristic of a quotient divisible group we mean the generalized cocharacteristic of its pseudo-rational envelope. Obviously, an embedding $\varphi: G \rightarrow \mathcal{R}(G)$ exists; for this reason, throughout the paper we shall identify the group $G$ and its image $\varphi(G)$.

Theorem 1 (9]). A quotient divisible group is the additive group of an $R$-module if and only if its p-adic completion is a finite group for any prime $p$.

Consider several statements proved in [5] and [7].

Theorem 2 ([5]). If $\left\{x_{1}, \ldots, x_{n}\right\}$ is a fundamental system of a quotient divisible group $G$, then $\mathcal{R}(G)=\left\langle x_{1}, \ldots, x_{n}\right\rangle_{R}$. 
Theorem 3 (5]). If $\left\{x_{1}, \ldots, x_{n}\right\}$ is an arbitrary linearly independent system of generators of an $R$-module $M$, then $G=\left\langle x_{1}, \ldots, x_{n}\right\rangle_{*} \subseteq M$ is a quotient divisible group, and $\left\{x_{1}, \ldots, x_{n}\right\}$ is its fundamental system.

Lemma 1 ([7]). Let $G$ and $H$ be some quotient divisible mixed groups; moreover, assume that either $H$ is a reduced group or $G$ is divisible. Let $\bigoplus_{i=1}^{n} \mathbb{Z} x_{i}$ be a fundamental subgroup of $G$, and let $\varphi: G \rightarrow H$ be an arbitrary homomorphism. If

$$
g=r_{1} x_{1}+\cdots+r_{n} x_{n} \in G, \quad r_{1}, \ldots, r_{n} \in R,
$$

then $\varphi(g)=r_{1} \varphi\left(x_{1}\right)+\cdots+r_{n} \varphi\left(x_{n}\right)$.

Theorem 4. Let $G$ and $H$ be quotient divisible groups, let $X=\left\{x_{1}, \ldots, x_{n}\right\}$ be a fundamental system of the group $G$, and let $\alpha, \beta \in \operatorname{Hom}(G, H)$. Then

$$
\alpha=\beta \Leftrightarrow \alpha\left(x_{i}\right)=\beta\left(x_{i}\right)
$$

for any $i \in\{1, \ldots, n\}$.

Proof. The implication " $\Rightarrow$ " is obvious. We prove " $\Leftarrow$ ".

The first case. The group $H$ is reduced. By Theorem 2, any element $g \in G$ can be represented in the form

$$
g=r_{1} x_{1}+\cdots+r_{n} x_{n}, \quad r_{1}, \ldots, r_{n} \in R .
$$

By Lemma 1,

$$
\alpha(g)=r_{1} \alpha\left(x_{1}\right)+\cdots+r_{n} \alpha\left(x_{n}\right)=r_{1} \beta\left(x_{1}\right)+\cdots+r_{n} \beta\left(x_{n}\right)=\beta(g),
$$

whence $\alpha=\beta$.

The second case. $H=\operatorname{div} H \oplus H_{1}$, where $\operatorname{div} H$ is divisible and $H_{1}$ is a reduced part of the group $H$. Then

$$
\operatorname{Hom}(G, H)=\operatorname{Hom}(G, \operatorname{div} H) \oplus \operatorname{Hom}\left(G, H_{1}\right),
$$

and $\alpha=\alpha_{1}+\alpha_{2}$ and $\beta=\beta_{1}+\beta_{2}$, where $\alpha_{1}, \beta_{1} \in \operatorname{Hom}(G$, $\operatorname{div} H)$ and $\alpha_{2}, \beta_{2} \in$ $\operatorname{Hom}\left(G, H_{1}\right)$. Since $\alpha(X)=\beta(X)$, we have $\alpha_{1}(X)=\beta_{1}(X)$ and $\alpha_{2}(X)=\beta_{2}(X)$. Then the first case implies that $\alpha_{2}=\beta_{2}$.

Let $g$ be an arbitrary element of the group $G$. Then the definition of a quotient divisible group shows that there exists a natural number $m$ such that $m g=m_{1} x_{1}+\cdots+m_{n} x_{n}$, where $m_{1}, \ldots, m_{n} \in \mathbb{Z}$. Since $\alpha_{1}(X)=\beta_{1}(X)$, we have

$$
\alpha_{1}(m g)=m_{1} \alpha_{1}\left(x_{1}\right)+\cdots+m_{n} \alpha_{1}\left(x_{n}\right)=m_{1} \beta_{1}\left(x_{1}\right)+\cdots+m_{n} \beta_{1}\left(x_{n}\right)=\beta_{1}(m g),
$$

whence $m\left(\alpha_{1}(g)-\beta_{1}(g)\right)=0$. But $\alpha_{1}(g)-\beta_{1}(g) \in \operatorname{div} H$, and $\operatorname{div} H$ contains no nonzero elements of finite order. Consequently, $\alpha_{1}(g)-\beta_{1}(g)=0$ and $\alpha_{1}(g)=\beta_{1}(g)$. Therefore, $\alpha_{1}=\beta_{1}$ and, thus, $\alpha=\alpha_{1}+\alpha_{2}=\beta_{1}+\beta_{2}=\beta$.

\section{§3. The module of PSEUdo-RAtional RElations}

Let $M$ be a finitely generated $R$-module, and $X=\left\{x_{1}, \ldots, x_{n}\right\}$ an arbitrary system of elements in $M$. Consider the set

$$
\Delta M_{X}=\left\{\left(r_{1}, \ldots, r_{n}\right) \in R^{n} \mid r_{1} x_{1}+\cdots+r_{n} x_{n}=0\right\},
$$

which is obviously an $R$-module. If $X$ is a system of generators in $M$, then $\Delta M_{X}$ will be called the module of pseudo-rational relations of $M$.

Proposition 1. If $X=\left\{x_{1}, \ldots, x_{n}\right\}$ is a system of generators of an $R$-module $M$, then $M \cong R^{n} / \Delta M_{X}$. 
Proof. Consider the mapping $\varphi: R^{n} \rightarrow M$ given by the rule

$$
\varphi\left(r_{1}, \ldots, r_{n}\right)=r_{1} x_{1}+\cdots+r_{n} x_{n} .
$$

It is easily seen that $\varphi$ is a homomorphism. Since $X$ is a system of generators of the $R$-module $M, \varphi$ is an epimorphism. Note that

$$
\left(r_{1}, \ldots, r_{n}\right) \in \operatorname{ker} \varphi \Leftrightarrow r_{1} x_{1}+\cdots+r_{n} x_{n}=0,
$$

i.e., $\operatorname{ker} \varphi=\Delta M_{X}$. Thus, $M \cong R^{n} / \Delta M_{X}$.

Corollary 1. If $X=\left\{x_{1}, \ldots, x_{n}\right\}$ is a system of generators of an $R$-module $M$, then $r^{*}(M)+r^{*}\left(\Delta M_{X}\right)=n$.

Let $G$ be a quotient divisible group, and let $X=\left\{x_{1}, \ldots, x_{n}\right\}$ be a system of its elements. Then $G$ gives rise to the $R$-module

$$
\Delta G_{X}=\left\{\left(r_{1}, \ldots, r_{n}\right) \in R^{n} \mid r_{1} x_{1}+\cdots+r_{n} x_{n} \in \operatorname{div} G\right\} .
$$

If $X$ is a fundamental system of the group $G$, then the $R$-module $\Delta G_{X}$ is called the module of pseudo-rational relations of the quotient divisible group $G$.

If $X=\left\{x_{1}, \ldots, x_{n}\right\}$ is a fundamental system of a quotient divisible group $G$, then $\mathcal{R}(G)=\left\langle x_{1}, \ldots, x_{n}\right\rangle_{R}$. So,

$$
\mathcal{R}(G) \cong \operatorname{div} G \oplus\left(R^{r(G)} / \Delta G_{X}\right) \quad \text { and } \quad r^{*}(G)=r(\operatorname{div} G)+r(G)-r^{*}\left(\Delta G_{X}\right) .
$$

Theorem 5 ([7]). Let $G$ and $H$ be arbitrary quotient divisible groups, $X=\left\{x_{1}, \ldots, x_{n}\right\}$ a fundamental system of $G$, and $Y=\left\{y_{1}, \ldots, y_{n}\right\}$ an arbitrary system of elements of $H$. A homomorphism $f: G \rightarrow H$ such that $f\left(x_{i}\right)=y_{i}(1 \leq i \leq n)$ exists if and only if $\Delta G_{X} \subseteq \Delta H_{Y}$.

If $\Delta G_{X}$ is the module of pseudo-rational relations of a quotient divisible group $G$ of rank $n$ and $A$ is an $(n \times n)$-matrix with integral entries, then we define the product $\Delta G_{X} \cdot A$ in the following way:

$$
\Delta G_{X} \cdot A=\left\{\left(r_{1}, \ldots, r_{n}\right) \cdot A \mid\left(r_{1}, \ldots, r_{n}\right) \in \Delta G_{X}\right\} .
$$

Lemma 2. If $X=\left\{x_{1}, \ldots, x_{n}\right\}$ and $Y=\left\{y_{1}, \ldots, y_{n}\right\}$ are arbitrary maximal linearly independent systems of a quotient divisible group $G$, then there exists a matrix $A \in$ $G L(n, \mathbb{Q})$ with integral entries such that $\Delta G_{X} \cdot A \doteq \Delta G_{Y}$.

Proof. Since $X$ and $Y$ are maximal systems, it follows that there are numbers $m, k \in \mathbb{Z}$ and matrices $A$ and $B$ with integral entries such that

$$
m\left(\begin{array}{c}
x_{1} \\
\cdots \\
x_{n}
\end{array}\right)=A\left(\begin{array}{c}
y_{1} \\
\cdots \\
y_{n}
\end{array}\right) \text { and } k\left(\begin{array}{c}
y_{1} \\
\cdots \\
y_{n}
\end{array}\right)=B\left(\begin{array}{c}
x_{1} \\
\cdots \\
x_{n}
\end{array}\right) .
$$

Let $\left(r_{1}, \ldots, r_{n}\right) \in \Delta G_{X}$. Then $\left(r_{1}, \ldots, r_{n}\right)[X] \in \operatorname{div} G$, where $[X]$ is the column of the elements $x_{1}, \ldots, x_{n}$, so that $m\left(r_{1}, \ldots, r_{n}\right)[X] \in \operatorname{div} G$. It follows that $\left(r_{1}, \ldots, r_{n}\right) A[Y] \in$ $\operatorname{div} G$. Then

$$
\Delta G_{X} \cdot A \subseteq \Delta G_{Y}
$$

Similarly,

$$
\Delta G_{Y} \cdot B \subseteq \Delta G_{X} .
$$

Relation (1) and the maximality of $X$ and $Y$ imply that

$$
A B=B A=k m E \text {. }
$$

Next, multiplying (3) by $A$ from the right, we get

$$
k m \Delta G_{Y} \subseteq \Delta G_{X} \cdot A .
$$


From (2) and (4) it follows that $\Delta G_{X} \cdot A \doteq \Delta G_{Y}$.

Theorem 6. Quotient divisible groups $G$ and $H$ are quasi-isomorphic if and only if for arbitrary fundamental systems $X$ and $Y$ in $G$ and $H$, respectively, there exists a matrix $A \in G L(n, \mathbb{Q})$ with integral entries such that $\Delta G_{X} \cdot A \doteq \Delta H_{Y}$.

Proof. Let $\frac{1}{m} \otimes \alpha: G \rightarrow H$ and $\frac{1}{k} \otimes \beta: H \rightarrow G$ be mutually inverse quasi-isomorphisms, and let

$$
\alpha([X])=[U]=A[Y], \quad \beta([Y])=[T]=B[X] .
$$

We assume that the homomorphisms $\alpha$ and $\beta$ are chosen in such a way that the matrices $A$ and $B$ have integral entries (this assumption can be imposed because $\frac{1}{m} \otimes \alpha=\frac{1}{s m} \otimes s \alpha$ and $s \alpha([X])=s[U]=s A[Y])$. Then

$$
\begin{aligned}
& \alpha(\beta([Y]))=\alpha(B[X])=B \alpha([X])=B A[Y], \\
& \beta(\alpha([X]))=\beta(A[Y])=A \beta([Y])=A B[X] .
\end{aligned}
$$

On the other hand, since $\frac{1}{m} \otimes \alpha$ and $\frac{1}{k} \otimes \beta$ are mutually inverse, we have

$$
\alpha \beta=m k \cdot i d_{H} \quad \text { and } \quad \beta \alpha=m k \cdot i d_{G} .
$$

Thus, $B A=A B=m k E$.

By Theorem $5, \Delta G_{X} \subseteq \Delta H_{U}$ and $\Delta H_{Y} \subseteq \Delta G_{T}$. Since $[U]=A[Y]$ and $[T]=B[X]$, we have $\Delta H_{U} \cdot A \subseteq \Delta H_{Y}$ and $\Delta G_{T} \cdot B \subseteq \Delta G_{X}$. This implies that

$$
\Delta G_{X} \cdot A \subseteq \Delta H_{Y} \quad \text { and } \quad \Delta H_{Y} \cdot B \subseteq \Delta G_{X} .
$$

Multiplying the second inclusion by $A$ from the right, we obtain $k m\left(\Delta H_{Y}\right) \subseteq \Delta G_{X} \cdot A$, which implies the quasirelation $\Delta G_{X} \cdot A \doteq \Delta H_{Y}$.

Conversely, assume that for quotient divisible groups $G$ and $H$ we have the quasirelation $\Delta G_{X} \cdot A \doteq \Delta H_{Y}$, where $A \in G L(n, \mathbb{Q})$ is a matrix with integral entries. Then $m\left(\Delta G_{X} \cdot A\right) \subseteq \Delta H_{Y}$ and $k\left(\Delta H_{Y}\right) \subseteq \Delta G_{X} \cdot A$ for some $m, k \in \mathbb{N}$. Let $[U]=m A[Y]$. If $\left(r_{1}, \ldots, r_{n}\right) \in \Delta G_{X}$, then

$$
\left(r_{1}, \ldots, r_{n}\right) m A[Y]=\left(r_{1}, \ldots, r_{n}\right)[U] \in \operatorname{div} H,
$$

so that $\Delta G_{X} \subseteq \Delta H_{U}$. By Theorem 5 , there exists a homomorphism $\alpha: G \rightarrow H$ such that $\alpha([X])=[U]=m A[Y]$.

Since the matrix $A$ lies in $G L(n, \mathbb{Q})$, there exists a matrix $B \in G L(n, \mathbb{Q})$ with integral entries such that $A B=B A=n E$, where $n$ is a nonzero integer. We multiply the quasirelation $\Delta G_{X} \cdot A \doteq \Delta H_{Y}$ by the matrix $B$ from the right obtaining

$$
\Delta H_{Y} \cdot B \doteq n \Delta G_{X} \doteq \Delta G_{X} .
$$

In the same way as above, we prove the existence of a homomorphism $\beta: H \rightarrow G$ such that $\beta([Y])=[T]=k B[X]$, where $k$ is a nonzero integer. We find $\alpha \beta([Y])$ :

$$
\alpha(\beta([Y]))=\alpha(k B[X])=k B \alpha([X])=k B m A[Y]=k m n[Y] .
$$

Similarly, we check that $\beta \alpha([X])=k m n[X]$. Then, by Theorem 4 ,

$$
\alpha \beta=k m n \cdot i d_{H} \quad \text { and } \quad \beta \alpha=k m n \cdot i d_{G} .
$$

Thus, the quasihomomorphisms $\frac{1}{m n} \otimes \alpha$ and $\frac{1}{k} \otimes \beta$ are mutually inverse; i.e., the groups $G$ and $H$ are quasi-isomorphic.

Lemma 2 and Theorem 6 imply the following statement. 
Corollary 2. Let $X=\left\{x_{1}, \ldots, x_{n}\right\}$ be a maximal linearly independent system of elements of a quotient divisible group $G$. Then the subgroup

$$
H=\left\langle x_{1}, \ldots, x_{n}\right\rangle_{*} \subseteq\left\langle x_{1}, \ldots, x_{n}\right\rangle_{R}
$$

of $G$ is a quotient divisible group quasi-isomorphic to $G$.

In conclusion of the present section, we touch on the problem concerning quasi-equality of $R$-modules in general and of modules of pseudo-rational relations in particular.

Proposition 2. $R$-modules $M$ and $L$ (not necessarily finitely generated) are quasi-equal if and only if $(1-\varepsilon) M=(1-\varepsilon) L$ and $\varepsilon M$ is quasi-equal to $\varepsilon L$, where $(1-\varepsilon)$ is an idempotent of the form (b) of the ring $R$.

Proof. If $M$ and $L$ are quasi-equal $R$-modules, then $M /(M \cap L)$ and $L /(M \cap L)$ are finite $R$-modules; therefore,

$$
(1-\varepsilon) M=(1-\varepsilon)(M \cap L)=(1-\varepsilon) L
$$

for some idempotent $(1-\varepsilon) \in R$ of the form (b).

Since the $R$-modules $M$ and $L$ are quasi-equal, the $R$-modules $\varepsilon M$ and $\varepsilon L$ are also quasi-equal.

The converse statement is obvious.

Let $G$ and $H$ be quasi-isomorphic quotient divisible groups. Then their modules of pseudo-rational relations are connected by the quasi-equality $\Delta G \cdot A \doteq \Delta H$, where $A$ is a nondegenerate matrix with integral entries (the form of which depends on the choice of fundamental systems in $G$ and $H$ ). Proposition 2 implies that

$$
(1-\varepsilon) \Delta G \cdot A=(1-\varepsilon) \Delta H \quad \text { and } \quad \varepsilon(\Delta G \cdot A) \doteq \varepsilon \Delta H .
$$

If $\varepsilon=\varepsilon_{p_{1}}+\cdots+\varepsilon_{p_{n}}$, then $\varepsilon \Delta G=\varepsilon_{p_{1}} \Delta G \oplus \cdots \oplus \varepsilon_{p_{n}} \Delta G$; consequently,

$$
\varepsilon(\Delta G \cdot A) \doteq \varepsilon \Delta H \Leftrightarrow \varepsilon_{p}(\Delta G \cdot A) \doteq \varepsilon_{p} \Delta H \text { for every } p \in\left\{p_{1}, \ldots, p_{n}\right\},
$$

and $\varepsilon_{p}(\Delta G \cdot A)$ and $\varepsilon_{p} \Delta H$ are finitely generated free $\widehat{\mathbb{Z}}_{p}$-modules for any prime $p \in$ $\left\{p_{1}, p_{2}, \ldots, p_{n}\right\}$.

\section{$\S 4$. The CATEgory $\mathfrak{T}$}

In this section, we translate the main results of the preceding section into the language of categories.

Let $M$ be a submodule of the free $R$-module $R^{n}$ such that $R^{n} / M$ is a reduced $R$ module. The set $M$ consists of some elements of the form $\left(r_{1}, \ldots, r_{n}\right) \in R^{n}$ and is closed under addition and multiplication by pseudo-rational numbers.

In the quotient module $R^{n} / M$, we consider the system of generators

$$
x_{1}=(1,0, \ldots, 0)+M, \quad x_{2}=(0,1, \ldots, 0)+M, \ldots, x_{n}=(0,0, \ldots, 1)+M .
$$

In a torsion-free divisible group $D$ of finite rank $n-r$, where $r$ is the number of elements in a maximal subsystem of $\left\{x_{1}, \ldots, x_{n}\right\}$ that is independent over $\mathbb{Z}$, we choose elements $d_{1}, \ldots, d_{n}$ in such a way that the elements

$$
x_{1}^{\prime}=d_{1}+x_{1}, \quad x_{2}^{\prime}=d_{2}+x_{2}, \ldots, x_{n}^{\prime}=d_{n}+x_{n}
$$

are linearly independent over $\mathbb{Z}$ in $D \oplus R^{n} / M$. Consider the group

$$
G=\left\langle x_{1}^{\prime}, \ldots, x_{n}^{\prime}\right\rangle_{*} \subseteq D \oplus R^{n} / M .
$$

Since $\left\{x_{1}^{\prime}, \ldots, x_{n}^{\prime}\right\}$ is a linearly independent (over $\mathbb{Z}$ ) system of generators of the $R$ module $D \oplus R^{n} / M$, the group $G$ is quotient divisible. 
Proposition 3. If $G$ is a quotient divisible group defined by the condition $(*)$, then $D \oplus$ $R^{n} / M$ is its pseudo-rational envelope and $M$ is its module of pseudo-rational relations.

Proof. Indeed, since $G \subseteq D \oplus R^{n} / M$ by construction, we have $\mathcal{R}(G) \subseteq D \oplus R^{n} / M$. Since the elements $x_{1}^{\prime}, \ldots, x_{n}^{\prime}$ generate the module $D \oplus R^{n} / M$ and are contained in $G$, it follows that $D \oplus R^{n} / M \subseteq \mathcal{R}(G)$. Thus, $D \oplus R^{n} / M$ is the pseudo-rational envelope of the group $G$.

Since the module $\Delta G_{X}$ is the kernel of the natural homomorphism from $R^{n}$ to $\mathcal{R}(G) / \operatorname{div} G=R^{n} / M$, we obtain $\Delta G_{X}=M$.

Let $\mathfrak{T}_{n}$ be the set of all submodules $M \subseteq R^{n}$ such that $R^{n} / M$ is a reduced $R$-module. We construct a category $\mathfrak{T}$, the objects of which are elements of the set $\bigcup_{n \in \mathbb{N}} \mathfrak{T}_{n}$. If $M_{1} \in \mathfrak{T}_{n}$ and $M_{2} \in \mathfrak{T}_{k}$ are arbitrary objects of $\mathfrak{T}$, then by a morphism of $M_{1}$ to $M_{2}$ we mean any $(n \times k)$-matrix $A$ with integral entries such that

$$
m M_{1} \cdot A \subseteq M_{2}
$$

for some natural $m$ (i.e., $M_{1} \cdot A$ is quasi-embedded in $M_{2}$ ). The product of morphisms $A: M_{1} \rightarrow M_{2}$ and $B: M_{2} \rightarrow M_{3}$ is the morphism $B A: M_{1} \rightarrow M_{3}$. Obviously, the identity matrix of size $n$ is the identity morphism of an object $M \in \mathfrak{T}_{n}$. It is easily seen that objects $M_{1}$ and $M_{2}$ of the category $\mathfrak{T}$ are isomorphic if and only if $M_{1}, M_{2} \in \mathfrak{T}_{n}$ for some $n \in \mathbb{N}$ and there exists a matrix $A$ in $G L(n, \mathbb{Q})$ with integral entries such that $M_{1} \cdot A \doteq M_{2}$.

We recall that $\mathcal{Q D}$ is the category of quotient divisible groups with quasihomomorphisms as morphisms.

Theorem 7. The catogories $\mathfrak{T}$ and $\mathcal{Q D}$ are equivalent.

Proof. We construct covariant functors $c: \mathfrak{T} \rightarrow \mathcal{Q D}$ and $d: \mathcal{Q D} \rightarrow \mathfrak{T}$ such that

$$
d \cdot c \cong i d_{\mathfrak{T}} \quad \text { and } \quad c \cdot d \cong i d_{\mathcal{Q D}},
$$

where $i d$ is the identity functor.

Let $M$ be an arbitrary object of the category $\mathfrak{T}$. Then we set $c(M)=G$, where $G$ is a quotient divisible group defined by the condition $(*)$.

Consider an arbitrary morphism $A$ of objects $M$ and $L$ in the category $\mathfrak{T}$. Let $G$ and $H$ be quotient divisible groups that correspond to the modules $M$ and $L$. Since $M=\Delta G_{X}$ and $L=\Delta H_{Y}$, we have $m \Delta G_{X} \cdot A \subseteq \Delta H_{Y}$ for some $m \in \mathbb{N}$. Then if $X=\left\{x_{1}, \ldots, x_{n}\right\}$ and $Y=\left\{y_{1}, \ldots, y_{k}\right\}$, then

$$
\left(r_{1}, \ldots, r_{n}\right) m A[Y] \in \operatorname{div} H
$$

for any $\left(r_{1}, \ldots, r_{n}\right) \in \Delta G_{X}$. Let $U=\left\{u_{1}, \ldots, u_{k}\right\}$ be a system of elements in $H$ such that $[U]=m A \cdot[Y]$; then $\Delta G_{X} \subseteq \Delta H_{U}$, and thus, by Theorem 5 , there exists a homomorphism $\alpha: G \rightarrow H$ such that $\alpha\left(x_{i}\right)=u_{i}$ for every $i \in\{1,2, \ldots, n\}$. We set $c(A)=\frac{1}{m} \otimes \alpha$ and verify that the quasihomomorphism $c(A)$ does not depend on the choice of the number $m$. Let $m_{1} \in \mathbb{N}$ be such that $m_{1} \Delta G_{X} \cdot A \subseteq \Delta H_{Y}$. Then the pair $\left(m_{1}, A\right)$ determines a homomorphism $\alpha_{1}: G \rightarrow H$ such that $\alpha_{1}([X])=m_{1} A[Y]$. Using the relation $\alpha([X])=m A[Y]$ and Theorem 4 , we see that $m_{1} \alpha=m \alpha_{1}$, whence

$$
\frac{1}{m m_{1}} \otimes m_{1} \alpha=\frac{1}{m m_{1}} \otimes m \alpha_{1},
$$

i.e., $\frac{1}{m} \otimes \alpha=\frac{1}{m_{1}} \otimes \alpha_{1}$.

Let $G$ and $H$ be arbitrary groups in the category $\mathcal{Q D}$, and let $X=\left\{x_{1}, \ldots, x_{n}\right\}$ and $Y=\left\{y_{1}, \ldots, y_{k}\right\}$ be fundamental systems in $G$ and $H$, respectively. Then we define

$$
d(G)=\Delta G_{X} \quad \text { and } \quad d(H)=\Delta H_{Y} .
$$


If $\frac{1}{m} \otimes \alpha: G \rightarrow H$ is a quasihomomorphism and $\alpha(X)=U$, then $\Delta G_{X} \subseteq \Delta H_{U}$ by Theorem 5. Since $Y$ is a fundamental system of the group $H$ and $U \subset H$, there exists an $(n \times k)$-matrix $A$ with integral entries and $s \in \mathbb{N}$ such that $s[U]=A[Y]$. Since $s \alpha([X])=s[U]=A[Y]$, it follows that

$$
\Delta G_{X} \subseteq \Delta H_{s U}=\Delta H_{A Y} .
$$

Suppose $\left(r_{1}, \ldots, r_{n}\right) \in \Delta H_{A Y}$. Then $\left(r_{1}, \ldots, r_{n}\right) A Y \in \operatorname{div} H$, so that

$$
\Delta H_{A Y} \cdot A \subseteq \Delta H_{Y} .
$$

Taken together, (2) and (3) imply $\Delta G_{X} \cdot A \subseteq \Delta H_{Y}$. Next, we define $d\left(\frac{1}{m} \otimes \alpha\right)=A$.

Theorem 6 shows that the functors constructed satisfy condition (1); i.e., the categories $\mathfrak{T}$ and $\mathcal{Q D}$ are equivalent.

\section{§5. Completely Decomposable quotient Divisible Groups}

By analogy with torsion-free groups, a quotient divisible group is said to be completely decomposable if it decomposes into a direct sum of quotient divisible groups of rank 1 , and it is almost completely decomposable if it contains a completely decomposable quotient divisible subgroup of finite index.

Theorem 8. A quotient divisible group is completely decomposable if and only if it has a fundamental system independent over $R$.

Proof. Let $G$ be a completely decomposable quotient divisible group, and let $G=A_{1} \oplus$ $\cdots \oplus A_{n}$ be its decomposition into a direct sum of groups of rank 1. For every term $A_{i}$ $(i \in\{1, \ldots, n\})$, we consider a fundamental element $x_{i}$. By Theorem 2, we have

$$
\mathcal{R}(G)=\mathcal{R}\left(A_{1}\right) \oplus \cdots \oplus \mathcal{R}\left(A_{n}\right)=\left\langle x_{1}\right\rangle_{R} \oplus \cdots \oplus\left\langle x_{n}\right\rangle_{R} .
$$

On the other hand, $\left\{x_{1}, \ldots, x_{n}\right\}$ is a fundamental system of the group $G$ and, thus, $\mathcal{R}(G)=\left\langle x_{1}, \ldots, x_{n}\right\rangle_{R}$. Consequently,

$$
\left\langle x_{1}, \ldots, x_{n}\right\rangle_{R}=\left\langle x_{1}\right\rangle_{R} \oplus \cdots \oplus\left\langle x_{n}\right\rangle_{R}
$$

i.e., the system $\left\{x_{1}, \ldots, x_{n}\right\}$ is independent over the $\operatorname{ring} R$.

Conversely, let $\left\{x_{1}, \ldots, x_{n}\right\}$ be a fundamental system of the group $G$ independent over $R$, and let $A_{i}=\left\langle x_{i}\right\rangle_{*}$ be a rank 1 quotient divisible subgroup of $G$ for every $i \in\{1, \ldots, n\}$. We show that $G=A_{1} \oplus \cdots \oplus A_{n}$.

Any element $g \in G$ can be represented in the form $g=r_{1} x_{1}+\cdots+r_{n} x_{n}$, where $r_{1}, \ldots, r_{n} \in R$. On the other hand, $m g=m_{1} x_{1}+\cdots+m_{n} x_{n}$ for some $m_{1}, \ldots, m_{n} \in \mathbb{Z}$ and $m \in \mathbb{N}$. Since $x_{1}, \ldots, x_{n}$ are independent over $R$, we have $m r_{i} x_{i}=m_{i} x_{i} \in\left\langle x_{i}\right\rangle$ for every $i \in\{1, \ldots, n\}$, whence $r_{i} x_{i} \in A_{i}$. It follows that $G=A_{1}+\cdots+A_{n}$.

If $g=a_{1}+\cdots+a_{n}$, where $a_{i} \in A_{i}$, then $a_{i}=s_{i} x_{i}\left(s_{i} \in R\right)$ for every $i \in\{1, \ldots, n\}$. Then $g=s_{1} x_{1}+\cdots+s_{n} x_{n}=r_{1} x_{1}+\cdots+r_{n} x_{n}$. But the elements $x_{1}, \ldots, x_{n}$ are independent over $R$, and consequently, $a_{i}=s_{i} x_{i}=r_{i} x_{i}$ for every $i \in\{1, \ldots, n\}$, i.e., $G=A_{1} \oplus \cdots \oplus A_{n}$.

Corollary 3. A quotient divisible group is almost completely decomposable if and only if it has an independent over $R$ maximal system linearly independent over $\mathbb{Z}$.

Example. Consider the $R$-module $M=R \oplus \widehat{\mathbb{Z}}_{p}$, in which we take the elements $x_{1}=$ $(1,0)$ and $x_{2}=\left(\varepsilon_{p}, \varepsilon_{p}\right)$. We use them to construct the quotient divisible group $G=$ $\left\langle x_{1}, x_{2}\right\rangle_{*} \subset M$. Since $x_{1}$ and $x_{2}$ are independent over $R$, it follows that the group $G$ decomposes into a direct sum of quotient divisible groups, $G=H \oplus K$. Moreover, $H=\left\langle x_{1}\right\rangle_{*} \subset M$ and $K=\left\langle x_{2}\right\rangle_{*} \subset M$, i.e., $H \cong \mathbb{Z}$ and $K \cong \mathbb{Q}_{p}$. 


\section{§6. Groups With Finitely Generated Modules of PSEUdo-RATional RELATiOnS}

Theorem 6 states that two quotient divisible groups $G$ and $H$ are quasi-isomorphic if and only if there exists a nondegenerate matrix $A$ with integral entries such that

$$
\Delta G_{X} \cdot A \doteq \Delta H_{Y}
$$

where $X$ and $Y$ are arbitrary fundamental systems in $G$ and $H$, respectively. Thus, we may say that a quotient divisible group is determined (up to quasi-isomorphism) by its module of pseudo-rational relations. In this connection, it is natural to consider the case where the module of pseudo-rational relations has a rather simple structure, namely, the case where it is finitely generated.

Let $G$ be a quotient divisible group with a finitely generated module $\Delta G_{X}$ of pseudorational relations, and let $r(G)=n$. Then its pseudo-rational envelope has the form $\mathcal{R}(G) \cong \operatorname{div} G \oplus R^{n} / \Delta G_{X}$, and the $R$-module $R^{n} / \Delta G_{X}$ is finitely representable. In [8], it was shown that a finitely representable $R$-module decomposes into the direct sum of a projective and a finite module. Also in [8, it was proved that a finitely generated projective $R$-module $M$ has the form

$$
M \cong(1-\varepsilon) R^{k} \oplus K_{\varphi_{1}} \oplus \cdots \oplus K_{\varphi_{s}},
$$

where $\varphi_{1}, \ldots, \varphi_{s}$ are almost zero idempotent characteristics.

Thus, the module $\mathcal{R}(G)$ has the form

$$
\mathcal{R}(G) \cong \operatorname{div} G \oplus(1-\varepsilon) R^{k} \oplus K_{\chi_{1}} \oplus \cdots \oplus K_{\chi_{m}},
$$

where $\chi_{1}, \ldots, \chi_{m}$ are some (not necessarily idempotent) almost zero characteristics. Consequently, the generalized cocharacteristic of the $R$-module $\mathcal{R}(G)$ and, thus, of the group $G$ has the form

$$
\rho_{1}, \ldots, \rho_{k}, \chi_{1}, \ldots, \chi_{m}, 0, \ldots, 0,
$$

where $\rho_{1}=\cdots=\rho_{k}=(1-\varepsilon) \rho$.

Theorem 9. A reduced quotient divisible group $G$ has a finitely generated module of pseudo-rational relations if and only if its generalized cocharacteristic has the form $\rho_{1}$, $\ldots, \rho_{k}, \chi_{1}, \ldots, \chi_{m}$, where the characteristics $\rho_{1}, \ldots, \rho_{k}$ differ from the characteristic $\rho=(\infty)$ by a finite number of $\rho$-components, the characteristics $\chi_{1}, \ldots, \chi_{m}$ are almost zero, and $k=r^{*}(G)$.

Proof. The "only if" statement follows from all we have said above. We prove the converse statement. Let a group $G$ have a generalized cocharacteristic $\rho_{1}, \ldots, \rho_{k}, \chi_{1}$, $\ldots, \chi_{m}$ satisfying the assumptions of the theorem. Consider the pseudo-rational envelope $\mathcal{R}(G)$ of the group $G$. Since the characteristics $\chi_{1}, \ldots, \chi_{m}$ are almost zero, there exists an idempotent $(1-\varepsilon)$ of the form (b) such that the $R$-module $(1-\varepsilon) \mathcal{R}(G)$ has the generalized cocharacteristic $(1-\varepsilon) \rho_{1}, \ldots,(1-\varepsilon) \rho_{k}$. Note that

$$
r^{*}((1-\varepsilon) \mathcal{R}(G))=r^{*}(\mathcal{R}(G))=k .
$$

By [9, Corollary 4.1], we have

$$
(1-\varepsilon) \mathcal{R}(G) \cong R_{(1-\varepsilon) \rho_{1}} \oplus \cdots \oplus R_{(1-\varepsilon) \rho_{k}} .
$$

The finitely generated $R$-module $\varepsilon \mathcal{R}(G)$ has pseudo-rational rank 0; applying [9, Corollary 3.1], we obtain

$$
\varepsilon \mathcal{R}(G) \cong K_{\varphi_{1}} \oplus \cdots \oplus K_{\varphi_{n}}
$$

where $\varphi_{1}, \ldots, \varphi_{n}$ are almost zero characteristics. From (1) and (2) it follows that the $R$-module $\mathcal{R}(G)$ can be represented in the form $P \oplus K$, where $P$ is a projective $R$-module and $K$ is a finite $R$-module. Then, by [8, Corollary 3], $\mathcal{R}(G)$ is a finitely representable 
$R$-module. Since $\mathcal{R}(G) \cong R^{r(G)} / \Delta G_{X}$, it follows that $\Delta G_{X}$ is a finitely generated $R$ module.

Corollary 6. If a quotient divisible group $G$ has a finitely generated module of pseudorational relations, then it can be represented in the form $G=G_{1} \oplus K$, where $G_{1}$ is a quotient divisible torsion-free group and $K$ is a finite group.

All the main results obtained above for quotient divisible groups can be carried over to torsion-free groups of finite rank. This follows from the duality constructed in [1 by Fomin and Wickless.

We recall that $\mathcal{Q D}$ is the category of quotient divisible groups with quasihomomorphisms as morphisms and $\mathcal{Q T} \mathcal{F}$ is the category of torsion-free groups of finite rank with quasihomomorphisms as morphisms.

Theorem 10 ([1]). The categories $\mathcal{Q T \mathcal { F }}$ and $\mathcal{Q D}$ are dual.

Corollary 7. The categories $\mathcal{Q T \mathcal { F }}$ and $\mathfrak{T}$ are dual.

If $f$ is the duality functor from $\mathcal{Q T \mathcal { F }}$ to $\mathcal{Q D}$, then two torsion-free groups of finite rank $G$ and $H$ are quasi-isomorphic if and only if the quotient divisible groups $f(G)$ and $f(H)$ are quasi-isomorphic.

In [5], the pseudo-rational type $\mathcal{R}(G)$ was defined for a torsion-free group $G$ of finite rank, and it was shown there that the $R$-modules $\mathcal{R}(G)$ and $\mathcal{R}(f(G))$ are quasiisomorphic. If $\chi_{1}, \ldots, \chi_{k}$ is the generalized cocharacteristic of the $R$-module $\mathcal{R}(G)$ (and thus, of the quotient divisible group $f(G)$ ), then the construction of the functor $f$ implies that the sequence of types $\left[\chi_{1}\right], \ldots,\left[\chi_{k}\right]$ is the Richman type of the group $G$.

Let $G$ be a torsion-free group of finite rank, $X=\left\{x_{1}, \ldots, x_{n}\right\}$ its maximal linearly independent system, $G^{*}=f(G)$ the quotient divisible group dual to $G$, and $X^{*}=$ $\left\{x_{1}^{*}, \ldots, x_{n}^{*}\right\}$ the fundamental system in $G^{*}$ dual to $X$. Then the $R$-module $\Delta G_{X^{*}}^{*}=$ $\Delta \mathcal{R}(G)_{X^{*}}$ is called the module of pseudo-rational relations of a torsion-free group $G$ of finite rank and is denoted by $\Delta G_{X}$. In [7, it was proved that

$$
\Delta G_{X}=\left\{\left(\varphi\left(x_{1}\right), \ldots, \varphi\left(x_{n}\right)\right) \mid \varphi \in \operatorname{Hom}(G, R)\right\} .
$$

Thus, we obtain the following theorem.

Theorem 11. Torsion-free groups $G$ and $H$ of finite rank are quasi-isomorphic if and only if there exists a matrix $A \in G L(n, \mathbb{Q})$ with integral entries such that

$$
\Delta G_{X} \cdot A \doteq \Delta H_{Y},
$$

where $X$ and $Y$ are some maximal linearly independent systems in the groups $G$ and $H$, respectively.

The pseudo-rational rank of a torsion-free group $G$ of finite rank is defined as the pseudo-rational rank of its pseudo-rational type

$$
r^{*}(G)=r^{*}(\mathcal{R}(G))=r^{*}\left(G^{*}\right) .
$$

Theorem 12. A torsion-free group $G$ of finite rank has a finitely generated module of pseudo-rational relations if and only if its Richman type has the form $\tau_{1}, \ldots, \tau_{k}, \sigma_{1}, \ldots$, $\sigma_{m}$, where the types $\tau_{1}, \ldots, \tau_{k}$ consist of characteristics that differ from the characteristic $\rho=(\infty)$ by a finite number of p-components, the types $\sigma_{1}, \ldots, \sigma_{m}$ are almost zero, and $k=r^{*}(G)$.

Theorem 12 implies that all groups of the form $A \oplus M$, where $A$ is an almost divisible group and $M$ is a minimax group, have finitely generated modules of pseudo-rational relations. We recall that a group $A$ is said to be almost divisible if $p A=A$ for almost all 
prime numbers $p$, and a torsion-free group $A$ of finite rank is minimax if it is an extension of a free group of finite rank with the help of a divisible periodic group of finite rank. In other words, $A$ is an almost divisible group if and only if its Richman type consists of types the characteristics of which differ from the characteristic $\rho=(\infty)$ only at a finite number of places, and this number is equal to $r^{*}(A)$ (it is easily seen that for an almost divisible group $A$ we have $r^{*}(A)=r(A)$ ). A torsion-free group $M$ of finite rank is minimax if and only if its Richman type consists of almost zero types.

\section{REFERENCES}

[1] A. A. Fomin and W. Wickless, Quotient divisible abelian groups, Proc. Amer. Math. Soc. 126 (1998), 45-52. MR 1443826 (98c:20100)

[2] R. Beaumont and R. Pierce, Torsion free rings, Illinois J. Math. 5 (1961), 61-98. MR0148706 $(26: 6212)$

[3] B. Jónsson, On direct decompositions of torsion-free abelian groups, Math. Scand. 5 (1957), 230-235; 7 (1959), 316-371. MR0108454 (21:7170) MR0122872(23:A204)

[4] A. V. Yakovlev, On the classification problem of torsion-free abelian groups of finite rank, Zap. Nauchn. Sem. Leningrad. Otdel. Mat. Inst. Steklov. (LOMI) 57 (1976), 171-175; English transl., J. Soviet Math. 11 (1979), no. 4, 660-663. MR0460488 (57:481)

[5] A. A. Fomin, Quotient divisible mixed groups, Abelian Groups. Rings and Modules (Perth, 2000), Contemp. Math., vol. 273, Amer. Math. Soc., Providence, RI, 2001, pp. 117-128. MR1817156 (2002b:20079)

[6] L. Fuchs, Infinite abelian groups. Vol. I, Pure Appl. Math., vol. 36, Acad. Press, New York-London, 1970; Vol. II, Pure Appl. Math., vol. 36-II, Acad. Press, New York-London, 1973. MR0255673 (41:333) MR0349869 (50:2362)

[7] A. V. Tsarev, The pseudo-rational rank of an abelian group, Sibirsk. Mat. Zh. 46 (2005), no. 1, 217-229; English transl., Siberian Math. J. 46 (2005), no. 1, 172-181. MR.2141315 (2006a:20099)

[8] _ Projective and generating modules over the ring of pseudo-rational numbers, Mat. Zametki 80 (2006), no. 3, 437-448; English transl., Math. Notes 80 (2006), no. 3-4, 417-427. MR2280008 (2007m:13013)

[9] Modules over the ring of pseudo-rational numbers and quotient divisible groups, Algebra i Analiz 18 (2006), no. 4, 198-214; English transl., St. Petersburg Math. J. 18 (2007), no. 4, 657-669. MR2262588 (2007g:20055)

Moscow State Pedagogical Institute, Russia

E-mail address: an-tsarev@yandex.ru

Received 27/NOV/2008

Translated by N. B. LEBEDINSKAYA 